
Not for reproduction, distribution or commercial use.

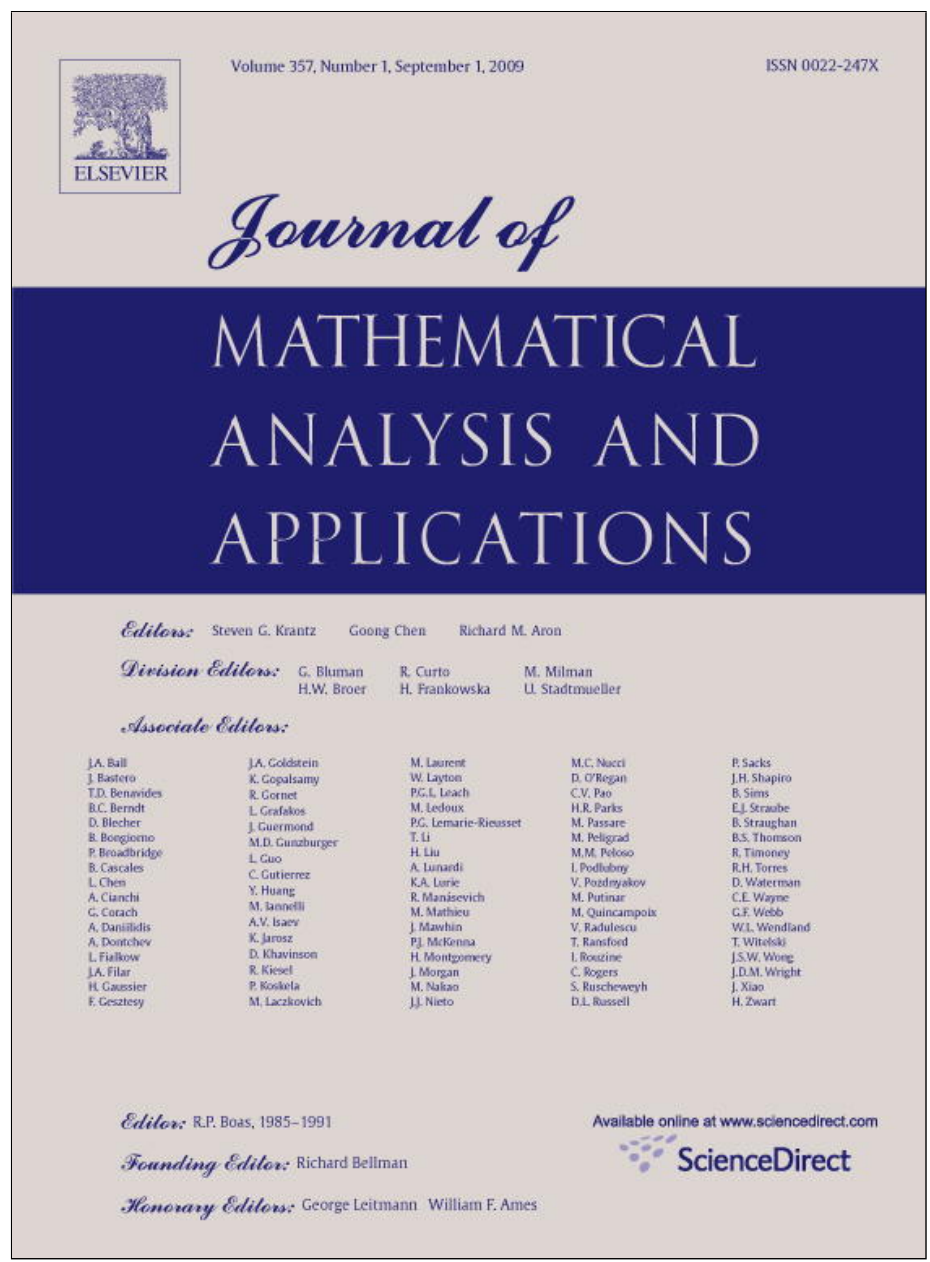

This article appeared in a journal published by Elsevier. The attached copy is furnished to the author for internal non-commercial research and education use, including for instruction at the authors institution and sharing with colleagues.

Other uses, including reproduction and distribution, or selling or licensing copies, or posting to personal, institutional or third party websites are prohibited.

In most cases authors are permitted to post their version of the article (e.g. in Word or Tex form) to their personal website or institutional repository. Authors requiring further information regarding Elsevier's archiving and manuscript policies are encouraged to visit:

http://www.elsevier.com/copyright 


\title{
Quadratic perturbations of quadratic codimension-four centers
}

\author{
Lubomir Gavrilov $^{\mathrm{a}, *}$, Iliya D. Iliev ${ }^{\mathrm{b}}$ \\ a Institut de Mathématiques de Toulouse, UMR 5219, Université Paul Sabatier (Toulouse III), 31062 Toulouse, Cedex 9, France \\ b Institute of Mathematics, Bulgarian Academy of Sciences, Bl. 8, 1113 Sofia, Bulgaria
}

\section{A R T I C L E I N F O}

\section{Article history:}

Received 26 November 2008

Available online 5 April 2009

Submitted by H.W. Broer

\section{Keywords:}

Quadratic codimension-four centers

Limit cycles

Zeros of Abelian integrals

\begin{abstract}
A B S T R A C T
We study the stratum in the set of all quadratic differential systems $\dot{x}=P_{2}(x, y), \dot{y}=$ $Q_{2}(x, y)$ with a center, known as the codimension-four case $Q_{4}$. It has a center and a node and a rational first integral. The limit cycles under small quadratic perturbations in the system are determined by the zeros of the first Poincaré-Pontryagin-Melnikov integral $I$. We show that the orbits of the unperturbed system are elliptic curves, and $I$ is a complete elliptic integral. Then using Picard-Fuchs equations and the Petrov's method (based on the argument principle), we set an upper bound of eight for the number of limit cycles produced from the period annulus around the center.
\end{abstract}

(c) 2009 Elsevier Inc. All rights reserved.

\section{Introduction}

The conditions for a plane polynomial quadratic vector field to have a center are known since the beginning of the last century (Dulac (1908), Kapteyn (1912)). In the space of all quadratic systems, the systems with a center form a union of four irreducible affine algebraic sets:

- Hamiltonian $\left(Q_{3}^{H}\right)$;

- reversible $\left(Q_{3}^{R}\right)$;

- generalized Lotka-Volterra $\left(Q_{3}^{L V}\right)$;

- codimension-four set $\left(Q_{4}\right)$

(the subscripts indicate the co-dimension of each algebraic subset), see Żołądek [11] for a modern proof. Strictly speaking, the above classification concerns only the centers themselves. There are quadratic systems with two centers, one of them in $Q_{3}^{R}$ and the other in $Q_{3}^{R} \cap Q_{3}^{L V}$, see [7, p. 148]. In the present paper we are interested in the maximal number of limit cycles which can emerge from the periodic orbits of a quadratic system with a center, after a small quadratic perturbation. The corresponding number is the cyclicity of the related open period annulus. A quadratic center is said to be generic, if it does not belong simultaneously to two of the above algebraic sets. The cyclicity of the period annulus of a generic center depends on the number of the zeros of the first Poincaré-Pontryagin-Melnikov function, while in the general case a higher-order analysis is needed. The corresponding higher-order functions were determined in Iliev [7].

The cyclicity of the open annuli in the generic Lotka-Volterra case $\left(Q_{3}^{L V}\right)$ has been studied by Żołądek [11], and in the generic Hamiltonian case $\left(Q_{3}^{H}\right)$ by Horozov-Iliev [6] and Gavrilov [4] (for the codimension-one generic cases from the bifurcation diagram of $Q_{3}^{H}$ see [1] and the references therein). Almost nothing is known about the generic reversible

\footnotetext{
* Corresponding author.

E-mail addresses: lubomir.gavrilov@math.univ-toulouse.fr (L. Gavrilov), iliya@math.bas.bg (I.D. Iliev).
} 
case $\left(Q_{3}^{R}\right)$ and nothing about the generic codimension-four one $\left(Q_{4}\right)$. In the present paper we place an upper bound on the cyclicity of the (unique) period annulus in the generic codimension-four case $\left(Q_{4}\right)$.

Theorem 1. The cyclicity of the open period annulus surrounding the center of any generic codimension-four plane quadratic system is less than or equal to eight.

The conjectural exact upper bound, as it is well known, is three [7,11]. To the end of this Introduction we briefly sketch our proof. A codimension-four plane quadratic system with a center has a well-known rational first integral $H$. Using this one may check that the generic complexified orbit $\left\{(x, y) \in \mathbb{C}^{2}: H(x, y)=h\right\}$ of the system is an affine elliptic curve, and the corresponding Poincaré-Pontryagin-Melnikov function $I=I(h)$ is a complete elliptic integral on it. This remarkable fact (it seems to have gone unnoticed by the specialists) is the starting point of the paper. Still, a direct application of standard techniques like in $[3,4,6,9,10]$ does not work. Indeed, the Poincaré-Pontryagin-Melnikov function involves differential of the third kind with residues algebraic in the parameter $h$ (and not only polynomial, as in the usual cases). On the other hand, it turns out that $I$ satisfies a Picard-Fuchs equation of the form

$$
M_{2} \circ L_{2} \circ L_{1}(I)=0
$$

in which $L_{1}=h \frac{d}{d h}-1$ and $L_{2}, M_{2}$ are suitable second-order Picard-Fuchs differential operators. The role of $L_{1}$ and $L_{2}$ in our approach is to annihilate the residues of the differentials involved in $I$. It is easy to see that $I$ has at most as many zeros as $G=L_{1}(I)$ on the interval of consideration. We shall prove further that the solution space of $L_{2}$ is a Chebyshev space (Proposition 14). This on its turn implies that if $R$ is a function with $k$ zeros, then each solution $G$ of the non-homogeneous equation $L_{2}(G)=R$ has at most $k+2$ zeros (Proposition 2). As $R=L_{2} \circ L_{1}(I)=L_{2}(G)$ is in the kernel of the second-order Picard-Fuchs operator $M_{2}$, it remains to show that $R$ has at most 6 zeros which is achieved in a standard way by making use of the Petrov method [10], see Proposition 4.

\section{Remarks about the codimension-four case}

In complex coordinate $z=x+i y$, the system corresponding to the generic codimension-four case with a center placed at the origin becomes

$$
\dot{z}=-i z+4 z^{2}+2|z|^{2}+\alpha \bar{z}^{2}, \quad \alpha \in \mathbb{C} \backslash \mathbb{R},|\alpha|=2 .
$$

It is well known that the codimension-four case has a first integral of the form

$$
\mathcal{H}(x, y)=\frac{[\varphi(x, y)]^{2}}{[\psi(x, y)]^{3}}
$$

where $\varphi$ and $\psi$ are polynomials of degree tree and two, respectively. Taking $\alpha=b+i c$ and denoting for short

$$
Y=c x-(2+b) y, \quad \kappa=\frac{4}{2+b}>1
$$

one can easily find the explicit form of the first integral, namely

$$
\mathcal{H}=\frac{\left[8 y(1+Y)-\frac{2}{3}\left(1+\kappa Y^{3}\right)\right]^{2}}{\left[1-8 y+\kappa Y^{2}\right]^{3}}
$$

Therefore, $\varphi=0$ defines a trident curve and $\psi=0$ is a parabola. Since $\varphi(0,0)=-\frac{2}{3}, \psi(0,0)=1$, the period annulus around the center is placed inside the domain $\Omega$ determined by $\varphi<0<\psi$. Taking $X^{2}=\psi=1-8 y+\kappa Y^{2}, X>0$, then in $(\bar{x}, \bar{y})=(X, Y)$ coordinates, the system has in $\Omega$ a first integral of the form

$$
H(\bar{x}, \bar{y})=\frac{\bar{x}^{-3}}{8(2-b)}\left(\frac{1}{3} \kappa \bar{y}^{3}+\kappa \bar{y}^{2}+\left(1-\bar{x}^{2}\right) \bar{y}-\bar{x}^{2}+\frac{1}{3}\right) .
$$

(Compare to [7], where an additional rotation of the very initial coordinates takes place.) Although the change of the variables

$$
(x, y) \mapsto(\bar{x}, \bar{y})
$$

does not define a bi-rational projective transformation (but a double ramified covering), it induces a bi-rational map

$$
\left\{(x, y): \frac{[\varphi(x, y)]^{2}}{[\psi(x, y)]^{3}}=t\right\} \rightarrow\{(\bar{x}, \bar{y}): H(\bar{x}, \bar{y})=t\} .
$$

It is seen that the level sets of the first integral $H(\bar{x}, \bar{y})$ are cubic plane curves and hence they are (generically) elliptic. 
For convenience, from now on, the bars over the variables $x, y$ will be omitted. The generating function $I(t)$, whose zeroes correspond to limit cycles in the perturbed system, are given by the following complete elliptic integral (cf. [7, Theorem 2(iii)])

$$
I(t)=\iint_{H(x, y)<t} x^{-6}\left[\mu_{1}+\mu_{2} y+\mu_{3} y^{3}+\mu_{4}\left(\kappa^{2} y^{4}-x^{4}\right)\right] d x d y .
$$

Our main purpose in this paper is to study how many zeroes the integral $I(t)$ can have in the open interval corresponding to the period annulus around $(1,0)$.

\section{Picard-Fuchs systems}

In this section we derive several equations satisfied by the entries of (2). We also apply several reductions to express these integrals in the form we need. In particular, we calculate explicitly $G, L_{2}$ and $R$ we mentioned in the introduction.

We begin by introducing new variables $y=y_{1}-1, h=8(2-b) t$. Then the equation $H(x, y)=t$ where $H$ is given by $(1)$ becomes (we will omit the subscript in $y_{1}$ )

$$
H(x, y, h)=\frac{\kappa}{3} y^{3}-x^{2} y-h x^{3}-(\kappa-1) y+\frac{2}{3}(\kappa-1)=0 .
$$

By using (3), it is not hard to verify that the period annulus around the center at $(1,1)$ corresponds to the levels $h$ from the interval $\left(-\frac{2}{3},-\frac{2}{3 \sqrt{\kappa}}\right)$.

Let us denote for $i, j \in \mathbb{Z}$

$$
I_{i, j}(h)=\iint_{H(x, y, h)<0} x^{i} y^{j} d x d y, \quad h \in\left(-\frac{2}{3},-\frac{2}{3 \sqrt{\kappa}}\right) .
$$

Then multiplying (3) by $x^{i} y^{j+1} d x$, respectively by $x^{i+1} y^{j} d y$ and integrating, we obtain the identities

$$
\begin{aligned}
& \frac{\kappa}{3}(j+4) I_{i, j+3}-(j+2) I_{i+2, j+1}-h(j+1) I_{i+3, j}-(\kappa-1)(j+2) I_{i, j+1}+\frac{2}{3}(\kappa-1)(j+1) I_{i, j}=0, \\
& \frac{\kappa}{3}(i+1) I_{i, j+3}-(i+3) I_{i+2, j+1}-h(i+4) I_{i+3, j}-(\kappa-1)(i+1) I_{i, j+1}+\frac{2}{3}(\kappa-1)(i+1) I_{i, j}=0 .
\end{aligned}
$$

Multiplying (4) by $i+4$ and (5) by $j+1$ and subtracting, we come to

$$
\kappa(i+j+5) I_{i, j+3}-(i+j+5) I_{i+2, j+1}-(\kappa-1)(i+3 j+7) I_{i, j+1}+2(\kappa-1)(j+1) I_{i, j}=0 .
$$

In particular, for $i=-6$ and $j=1$ one obtains $I_{-6,2}=I_{-6,1}$. Therefore, the function in (2) takes the form

$$
I(h)=\mu_{1} I_{-6,0}+\mu_{2} I_{-6,1}+\mu_{3} I_{-6,3}+\mu_{4}\left(\kappa^{2} I_{-6,4}-I_{-2,0}\right)
$$

with all constants $\mu_{i} \in \mathbb{R}$ independent.

Let us apply to (3) and (6) the change of variables $(x, y) \rightarrow\left(x^{-1}, y x^{-1}\right)$. Then (3) reduces to

$$
H(x, y) \equiv \frac{2}{3}(\kappa-1) x^{3}-(\kappa-1) x^{2} y+\frac{\kappa}{3} y^{3}-y=h,
$$

$I_{i, j}(h)$ becomes $-I_{-i-j-3, j}(h)$ and $(6)$ becomes

$$
I(h)=\iint_{H(x, y)<h}\left(\mu_{1} x^{3}+\mu_{2} x^{2} y+\mu_{3} y^{3}+\mu_{4}\left(\frac{\kappa^{2} y^{4}-1}{x}\right)\right) d x d y .
$$

By (7), $H(x, y)=-H(-x,-y)$, therefore the phase portrait of the related Hamiltonian system has a central symmetry with respect to the origin.

Next, we can use the following identities:

$$
\begin{aligned}
& I_{1,2}=I_{2,1}=\frac{3 h}{10} I_{0,0}+I_{1,0}+\frac{1}{5} I_{0,1}, \\
& I_{3,0}=\frac{3 \kappa h}{10(\kappa-1)} I_{0,0}+I_{1,0}+\frac{\kappa}{5(\kappa-1)} I_{0,1}, \\
& I_{0,3}=\frac{3(\kappa+1) h}{10 \kappa} I_{0,0}+\frac{\kappa-1}{\kappa} I_{1,0}+\frac{\kappa+6}{5 \kappa} I_{0,1}, \\
& I_{-1,4}=\frac{6 h}{5 \kappa} I_{-1,1}+\frac{9}{5 \kappa^{2}} I_{-1,0}+\frac{9(\kappa-1)}{5 \kappa^{2}} I_{1,0}+\frac{\kappa-1}{\kappa} I_{1,2}
\end{aligned}
$$


to transform (8) into

$$
I(h)=\mu_{1} h I_{0,0}(h)+\mu_{2} I_{1,0}(h)+\mu_{3} I_{0,1}(h)+\mu_{4}\left[2 I_{-1,0}(h)+3 \kappa h I_{-1,1}(h)\right] .
$$

Following the standard way [6], one can derive a Picard-Fuchs system for the entries in (9). Its explicit form is as follows.

$$
\begin{aligned}
& I_{0,0}=\frac{3 h}{2} I_{0,0}^{\prime}+I_{0,1}^{\prime}, \\
& I_{1,0}=h I_{1,0}^{\prime}+\frac{2}{3} I_{1,1}^{\prime}, \\
& I_{0,1}=\frac{2}{3 \kappa} I_{0,0}^{\prime}+h I_{0,1}^{\prime}+\frac{2(\kappa-1)}{3 \kappa} I_{1,1}^{\prime}, \\
& I_{1,1}=\frac{3 h}{8} I_{0,0}^{\prime}+\frac{1}{2} I_{1,0}^{\prime}+\frac{1}{4} I_{0,1}^{\prime}+\frac{3 h}{4} I_{1,1}^{\prime}, \\
& I_{-1,0}=3 h I_{-1,0}^{\prime}+2 I_{-1,1}^{\prime}, \\
& I_{-1,1}=\frac{\kappa-1}{\kappa} I_{1,0}^{\prime}+\frac{1}{\kappa} I_{-1,0}^{\prime}+\frac{3 h}{2} I_{-1,1}^{\prime} .
\end{aligned}
$$

By using the above system, we see that

$$
h I^{\prime}-I=G(h), \quad G(h)=\left(\mu_{1} h^{2}+\mu_{3}\right) I_{0,0}^{\prime}+\mu_{2} I_{1,1}^{\prime}+\mu_{4}\left[-4 h I_{-1,0}^{\prime}+\left(3 \kappa h^{2}-4\right) I_{-1,1}^{\prime}\right] .
$$

Therefore

$$
I(h)=h \int_{-\frac{2}{3}}^{h} \xi^{-2} G(\xi) d \xi
$$

and $I(h)$ has at most as much zeroes as $G(h)$ in $\left(-\frac{2}{3},-\frac{2}{3 \sqrt{\kappa}}\right)$. By the same system, the integrals $I_{0,0}^{\prime}$ and $I_{1,1}^{\prime}$ satisfy

$$
\begin{aligned}
& -3 \kappa h I_{0,0}^{\prime}=\left(9 \kappa h^{2}-4\right) I_{0,0}^{\prime \prime}-4(\kappa-1) I_{1,1}^{\prime \prime}, \\
& -3 \kappa h I_{1,1}^{\prime}=\left(9 \kappa h^{2}-4\right)\left(I_{0,0}^{\prime \prime}-I_{1,1}^{\prime \prime}\right),
\end{aligned}
$$

and the integrals $I_{-1,0}^{\prime}$ and $I_{-1,1}^{\prime}$ satisfy

$$
\begin{aligned}
& I_{-1,0}^{\prime}=-\frac{3 h}{2} I_{-1,0}^{\prime \prime}-I_{-1,1}^{\prime \prime}, \\
& I_{-1,1}^{\prime}=-\frac{2}{\kappa} I_{-1,0}^{\prime \prime}-3 h I_{-1,1}^{\prime \prime}+\frac{4(\kappa-1)}{3 \kappa h} I_{1,1}^{\prime \prime} .
\end{aligned}
$$

Hence, the integral $J=-4 h I_{-1,0}^{\prime}+\left(3 \kappa h^{2}-4\right) I_{-1,1}^{\prime}$ satisfies the second-order equation

$$
L_{2}(h) J=\frac{4}{3}(\kappa-1)\left[h\left(9 \kappa h^{2}-4\right) I_{1,1}^{\prime \prime \prime}+\left(6 \kappa h^{2}+8\right) I_{1,1}^{\prime \prime}\right]
$$

with

$$
L_{2}(h)=5 \kappa h-\left(9 \kappa h^{2}-8\right) \frac{d}{d h}+h\left(9 \kappa h^{2}-4\right) \frac{d^{2}}{d h^{2}} .
$$

Therefore, a similar equation $L_{2}(h) G=R$ (with a right-hand side $R$ depending linearly on $\mu_{i}, I_{0,0}^{\prime}$ and $I_{1,1}^{\prime}$ ) also holds. To calculate $R$ explicitly, we first use (10) to obtain the identities

$$
\begin{aligned}
& I_{0,0}^{\prime \prime}=\frac{-3 h\left(9 \kappa h^{2}-4\right) I_{0,0}^{\prime}+12(\kappa-1) h I_{1,1}^{\prime}}{\left(9 h^{2}-4\right)\left(9 \kappa h^{2}-4\right)}, \\
& I_{1,1}^{\prime \prime}=\frac{-3 h I_{0,0}^{\prime}+3 h I_{1,1}^{\prime}}{9 h^{2}-4}, \\
& I_{0,0}^{\prime \prime \prime}=\frac{324 \kappa h^{4}+(72 \kappa-108) h^{2}-48}{\left(9 h^{2}-4\right)^{2}\left(9 \kappa h^{2}-4\right)} I_{0,0}^{\prime}-\frac{12(\kappa-1)\left[243 \kappa h^{4}-36(\kappa+1) h^{2}-16\right]}{\left(9 h^{2}-4\right)^{2}\left(9 \kappa h^{2}-4\right)^{2}} I_{1,1}^{\prime}, \\
& I_{1,1}^{\prime \prime \prime}=\frac{27 h^{2}+12}{\left(9 h^{2}-4\right)^{2}} I_{0,0}^{\prime}-\frac{162 \kappa h^{4}+(144 \kappa-108) h^{2}-48}{\left(9 h^{2}-4\right)^{2}\left(9 \kappa h^{2}-4\right)} I_{1,1}^{\prime} .
\end{aligned}
$$

A direct calculation then yields

$$
R(h)=\frac{h\left[\left(a_{0}+a_{1} h^{2}+a_{2} h^{4}+a_{3} h^{6}\right) I_{0,0}^{\prime}+\left(b_{0}+b_{1} h^{2}+b_{2} h^{4}\right) I_{1,1}^{\prime}\right]}{\left(9 h^{2}-4\right)^{2}\left(9 \kappa h^{2}-4\right)},
$$


with some constants $a_{j}, b_{j}$ depending linearly on $\mu_{i}$. Below, we shall use the explicit formulas for $G, R, L_{2}$ just derived in order to prove our main result.

\section{Proof of Theorem 1}

The proof of Theorem 1 follows from the next four propositions, the first two of them being probably known. Let $V$ be a finite-dimensional vector space of functions, real-analytic on an open interval $(a, b)$.

Definition 1. We say that $V$ is a Chebyshev space, provided that each non-zero function in $V$ has at most $\operatorname{dim}(V)-1$ zeros, counted with multiplicity.

Let $S$ be the solutions space of a second-order linear analytic differential equation

$$
x^{\prime \prime}+a_{1}(t) x^{\prime}+a_{2}(t) x=0
$$

on an open interval $(a, b)$.

Proposition 1. The solution space $S$ of (12) is a Chebyshev space on the interval $(a, b)$ if and only if there exists a nowhere vanishing solution $x_{0} \in S\left(x_{0}(t) \neq 0, \forall t \in(a, b)\right)$.

Remark 1. The question of existence of a non-vanishing solution is a recurrent question in many papers concerning zeros of Abelian integrals, see e.g. [2,5,9]. A Chebyshev space $V$ in our sense is sometimes called an extended Chebyshev space, and it is said to be an extended complete Chebyshev space, provided that it has a complete flag of extended Chebyshev sub-spaces, see e.g. [8]. In the case when $\operatorname{dim}(V)=2$ the Chebyshev space $V$ (in our sense) is an extended complete Chebyshev one if and only if it has a nowhere vanishing function. Therefore the notions of Chebyshev space (in our sense) and extended complete Chebyshev space (in the sense of [8]), as far as applied to the solution space of (12), coincide.

Proposition 2. Suppose the solution space of the homogeneous equation (12) is a Chebyshev space and let $R$ be an analytic function on $(a, b)$ having $k$ zeros (counted with multiplicity). Then every solution $x(t)$ of the non-homogeneous equation

$$
x^{\prime \prime}+a_{1}(t) x^{\prime}+a_{2}(t) x=R(t)
$$

has at most $k+2$ zeros on $(a, b)$.

Proposition 3. The solution space $S$ associated to the differential operator

$$
L_{2}(h)=5 \kappa h-\left(9 \kappa h^{2}-8\right) \frac{d}{d h}+h\left(9 \kappa h^{2}-4\right) \frac{d^{2}}{d h^{2}}, \quad \kappa>1,
$$

is a Chebyshev system on the interval $\left(-\infty,-\frac{2}{3 \sqrt{\kappa}}\right)$.

Let $R=L_{2} \circ L_{1}(I)$, where $I$ is the Abelian integral (9) and $L_{1}=h \frac{d}{d h}-1$. A suitable for our purposes expression for $R$ is obtained in (11).

Proposition 4. The Abelian integral $R(h)$ has at most 6 zeros (counted with multiplicity) on the interval $\left(-\frac{2}{3},-\frac{2}{3 \sqrt{\kappa}}\right), \kappa>1$.

Proof of Theorem 1, assuming Propositions 1-4. The Abelian integral $L_{1}(I), I$ given by (9), is a solution of the nonhomogeneous equation $L_{2}(G)=R$. According to Proposition 4 , Proposition 3 and Proposition 2 the integral $L_{1}(I)(h)$ has at most 8 zeros on the interval $\left(-\infty,-\frac{2}{3 \sqrt{\kappa}}\right)$. The integral $I(h)$ has the same number of zeros as $L_{1}(I)(h)$ on the same interval. Finally, the functions (9), (8), (6) and (2) have the same number of zeros in the respective intervals.

To the end of the paper we prove the above Propositions 1-4.

Proof of Proposition 1. Let $\left(x_{1}, x_{2}\right)$ be a fundamental set of solutions of (12) and consider the map

$$
p:(a, b) \rightarrow S^{1}=\mathbb{P}^{1} \mathbb{R}: \quad t \mapsto\left[x_{1}(t): x_{2}(t)\right] .
$$

As the Wronskian of $x_{1}, x_{2}$ is non-vanishing, then the map $p$ is non-degenerate $(d p(t) \neq 0)$ and hence monotonous.

The solution space of (12) is Chebyshev if and only if the map $p$ is injective. The solution space of (12) contains a nowhere vanishing solution if and only if the map $p$ is not surjective.

As the circle is not homeomorphic to an open interval, then the monotonous differentiable map $p$ cannot be surjective and injective at the same time. It follows that if $p$ is injective then it is not surjective. If, on the contrary, $p$ is not surjective, then the monotonicity of $p$ implies that the image of $(a, b)$ under $p$ is an open subinterval of $S^{1}$ and $p$ is injective. 
Proof of Proposition 2. Let $\left(x_{1}, x_{2}\right)$ be a fundamental set of solutions of (12), such that $x_{1}(t)$ is a nowhere vanishing solution. The change of the variables $x \rightarrow x / x_{1}(t)$ does not change the number of the zeros of the solutions of (12), which is transformed to a linear equation with a fundamental system of solutions $\left\{1, \frac{x_{2}(t)}{x_{1}(t)}\right\}$. As the vector space spanned by $x_{1}, x_{2}$ is Chebyshev, then the function $\frac{x_{2}(t)}{x_{1}(t)}$ is strictly monotonous on $(a, b)$. The change of the independent variable $t \rightarrow \tau=\frac{x_{2}(t)}{x_{1}(t)}$ is therefore regular and transforms further the above linear equation to an equation with a fundamental system of solutions $\{1, \tau\}$. Therefore the corresponding differential operator is a multiple of $\frac{d^{2}}{d \tau^{2}}$. More precisely, the regular change of variables

$$
(x, t) \mapsto(y, \tau), \quad y=\frac{x}{x_{1}(t)}, \quad \tau=\frac{x_{2}(t)}{x_{1}(t)}
$$

transforms Eq. (13) to

$$
x_{1}\left(\frac{d}{d t} \frac{x_{2}(t)}{x_{1}(t)}\right)^{2} \frac{d^{2}}{d \tau^{2}} y=R(t(\tau))
$$

and hence each solution of the non-homogeneous equation (13) has at most $k+2$ zeros on $(a, b)$ (counted with multiplicity).

Proof of Proposition 3. Let $\left\{\delta(h): h \in\left(-\frac{2}{3},-\frac{2}{3 \sqrt{\kappa}}\right)\right\}$ be the continuous family of periodic orbits defined by $\{H=h\}$, with $H$ in the form (7). Then $G(h)=\int_{\delta(h)} \omega$ where $\omega$ is a linear combination of elliptic differentials of the first and second kind. Therefore the residues of $\omega$ are solutions of $L_{2}$. The only residues of $\omega$ are at $(0, y)$ where $y$ is one of the roots of $\frac{\kappa}{3} y^{3}-y=h$ and they are easily computed:

$$
\operatorname{Res}_{(0, y)} \omega=\frac{-4 h+\left(3 \kappa h^{2}-4\right) y}{\kappa y^{2}-1} .
$$

For $h<-\frac{2}{3 \sqrt{\kappa}}$ the polynomial $\frac{\kappa}{3} y^{3}-y-h$ has one real root which we denote by $y_{0}$. We shall show that the solution $f(h)=$ $\operatorname{Res}_{\left(0, y_{0}\right)} \omega$ of $L_{2} x=0$ does not vanish in the interval $\left(-\infty,-\frac{2}{3 \sqrt{\kappa}}\right)$. Indeed, on this interval $y_{0}(h)$ is a strictly increasing function and $y_{0}(h)<-\sqrt{\frac{5}{\kappa}}=y_{0}\left(-\frac{2}{3 \sqrt{\kappa}}\right)$. It remains to show that $-4 h+\left(3 \kappa h^{2}-4\right) y_{0} \neq 0$. The identity

$$
-4 h+\left(3 \kappa h^{2}-4\right) y_{0}=-4\left(\frac{\kappa}{3} y_{0}^{3}-y_{0}\right)+\left(3 \kappa h^{2}-4\right) y_{0}=\kappa y_{0}\left(3 h^{2}-\frac{4}{3} y_{0}^{2}\right)
$$

implies that $f(h)=0$ on $\left(-\infty,-\frac{2}{3 \sqrt{\kappa}}\right)$ if and only if $h=2 y_{0} / 3$. Now

$$
\frac{\kappa}{3} y_{0}^{3}-y_{0}=h=\frac{2}{3} y_{0}
$$

gives $y_{0}= \pm \sqrt{\frac{5}{\kappa}}$. But $y_{0}=y_{0}(h)$ is a strictly increasing function in $\left(-\infty,-\frac{2}{3 \sqrt{\kappa}}\right]$ and $y_{0}\left(-\frac{2}{3 \sqrt{\kappa}}\right)=-\sqrt{\frac{5}{\kappa}}$ which is the needed contradiction. Thus the solution space of $L$ on $\left(-\infty,-\frac{2}{3 \sqrt{\kappa}}\right)$ contains a nowhere vanishing function and hence is a Chebyshev system.

The above result cannot be improved, as shown by the example $L=\frac{d^{2}}{d t^{2}}$.

Proof of Proposition 4. According to (11), it suffices to show that any linear combination of the form $P_{3}\left(h^{2}\right) I_{0,0}^{\prime}(h)+$ $Q_{2}\left(h^{2}\right) I_{1,1}^{\prime}(h)$ where $P_{3}, Q_{2}$ are real polynomials of degree at most three and two, has at most 6 zeros. We note that $I_{0,0}^{\prime}, I_{1,1}^{\prime}$ are complete elliptic integrals of the first and second kind respectively, satisfying the second-order Picard-Fuchs system (10).

We introduce a new variable $s \in(1, \kappa)$ through $h=-\frac{2}{3} \sqrt{s / \kappa}$ and denote by dot the differentiation with respect to $s$. Also, denote for a convenience $J_{1}(s)=I_{0,0}^{\prime}(h(s)), J_{2}(s)=I_{1,1}^{\prime}(h(s))$. By (14) and (11), we obtain the equation

$$
L_{2} G \equiv\left[s(1-s) \frac{d^{2}}{d s^{2}}-\frac{1}{2} \frac{d}{d s}-\frac{5}{36}\right] G(s)=\frac{P_{3}(s) J_{1}(s)+Q_{2}(s) J_{2}(s)}{(s-\kappa)^{2}(s-1)} .
$$

Hence, we will need information about the zeroes of the right-hand side in the interval $(1, \kappa) \subset(1, \infty)$. Eq. $(10)$ implies that $J(s)=\left(J_{1}(s), J_{2}(s)\right)^{\top}$ satisfies the system of hypergeometric type

$$
J(s)=6\left(\begin{array}{cc}
1-s & \kappa-1 \\
1-s & s-1
\end{array}\right) \dot{J}(s)
$$


or equivalently

$$
6(s-1)(s-\kappa) \dot{J}(s)=\left(\begin{array}{cc}
1-s & \kappa-1 \\
1-s & s-1
\end{array}\right) J(s) .
$$

Let us consider for any $n \in \mathbb{N}$ the vector space

$$
V_{n}=\left\{P_{n} \cdot J_{1}+Q_{n-1} \cdot J_{2}: P_{n}, Q_{n-1} \in \mathbb{R}[h], \operatorname{deg} P_{m}, Q_{m} \leqslant m\right\} .
$$

Proposition 4 follows from the following more general result.

Proposition 5. The vector space $V_{n}$ is Chebyshev on the interval $(1, \kappa)$ : each element has at most $\operatorname{dim} V_{n}-1=2 n$ zeros (counted with multiplicity).

Proof of Proposition 5. We use the Petrov method in the complex domain $\mathcal{D}=\mathbb{C} \backslash(-\infty, 1)$, see $[9,10]$. The characteristic exponents of $(15)$ at $1, \kappa, \infty$ are equal to $\{0,0\},\{0,0\},\left\{-\frac{1}{6}, \frac{1}{6}\right\}$, respectively. The function $P_{n} . J_{1}+Q_{n-1} \cdot J_{2}$ is holomorphic in a neighborhood of $s=\kappa$ (this value corresponds to the center of the system $d H=0$, with $H$ the symmetric Hamiltonian given by (7)), and has a logarithmic singularity in a neighborhood of $s=1$ (which corresponds to the saddle point of the symmetric Hamiltonian system). The function $J_{1}$ is a complete elliptic integral of the first kind and therefore does not vanish. Consider the function

$$
F(s)=\frac{P_{n}(s) \cdot J_{1}(s)+Q_{n-1}(s) \cdot J_{2}(s)}{J_{1}(s)}
$$

which is real-analytic in the complex domain $\mathcal{D}$. We apply the argument principle to the smaller domain

$$
\mathcal{D}_{\varepsilon}=\mathcal{D} \cap\{s:|s-1|>\varepsilon\} \cap\left\{s:|s|<\frac{1}{\varepsilon}\right\} .
$$

For this purpose, we consider the increase (or decrease) of the argument of $F$ when $s$ makes one turn along the boundary of $\mathcal{D}_{\varepsilon}$ in a positive direction. The following facts are easily deduced from the asymptotic expansions of $J$ near the singular points of the Fuchs system (15).

1. Along the boundary of the small disc $\{|s-1|=\varepsilon\}$ the increase of the argument of $F$ is bounded by a value close to zero.

2. Along the boundary of the big disc $\left\{|s|=\frac{1}{\varepsilon}\right\}$ the increase of the argument of $F$ is bounded by a value close to $2 \pi n=$ $2 \pi \max \left\{n, n-1+\frac{2}{6}\right\}$.

3. Along the interval $(-\infty, 1)$, the imaginary part of $F$ equals

$$
Q_{n-1}(s) \operatorname{Im} \frac{J_{2}(s)}{J_{1}(s)}=Q_{n-1}(s) \frac{\operatorname{det} W(s)}{\left|J_{1}(s)\right|^{2}}
$$

where

$$
W(s)=\left(\begin{array}{ll}
J_{1}(s) & \tilde{J}_{1}(s) \\
J_{2}(s) & \tilde{J}_{2}(s)
\end{array}\right)
$$

is a fundamental matrix of (15).

4. The determinant of the fundamental matrix $W$ is a rational function in $s$ and in fact a non-zero constant.

Summing up the above facts we conclude that the increase of the argument of $F$ along the boundary of $\mathcal{D}_{\varepsilon}$ is bounded by $2 n$. Therefore $F$, and hence $P_{n}(s) . J_{1}(s)+Q_{n-1}(s) \cdot J_{2}(s)$ has at most $2 n$ zeros (counted with multiplicity) in $\mathcal{D}$, and hence in $(1, \kappa)$. Proposition 5 , and hence Proposition 4 are proved.

This also finishes the proof of Theorem 1 .

\section{Acknowledgments}

Part of the paper was written while the second author was visiting the University of Toulouse. He thanks for its hospitality. This research has been partially supported by PAI Rila program through Grants 14749SM (France) and Rila 3/6-2006 (Bulgaria).

\section{References}

[1] Shui-Nee Chow, Chengzhi Li, Yingfei Yi, The cyclicity of period annuli of degenerate quadratic Hamiltonian systems with elliptic segment loops, Ergodic Theory Dynam. Systems 22 (2) (2002) 349-374.

[2] W.A. Coppel, L. Gavrilov, The period function of a Hamiltonian quadratic system, Differential Integral Equations 6 (1993) $1357-1365$.

[3] L. Gavrilov, Abelian integrals related to Morse polynomials and perturbations of plane Hamiltonian vector fields, Ann. Inst. Fourier (Grenoble) 49 (1999) $611-652$. 
[4] L. Gavrilov, The infinitesimal 16th Hilbert problem in the quadratic case, Invent. Math. 143 (2001) 449-497.

[5] L. Gavrilov, I.D. Iliev, Complete hyperelliptic integrals of the first kind and their non-oscillation, Trans. Amer. Math. Soc. 356 (2004) 1185-1207.

[6] E. Horozov, I.D. Iliev, On the number of limit cycles in perturbations of quadratic Hamiltonian systems, Proc. London Math. Soc. 69 (1994) 198-224.

[7] Iliya D. Iliev, Perturbations of quadratic centers, Bull. Sci. Math. 122 (2) (1998) 107-161.

[8] S. Karlin, W. Studden, Tchebisheff Systems: With Applications in Analysis and Statistics, Interscience Publishers, 1966.

[9] G.S. Petrov, Elliptic integrals and their non-oscillation, Funct. Anal. Appl. 20 (1) (1986) 46-49 (in Russian).

[10] G.S. Petrov, Nonoscillation of elliptic integrals, Funct. Anal. Appl. 24 (3) (1990) 45-50 (in Russian).

[11] H. Żołądek, Quadratic systems with center and their perturbations, J. Differential Equations 109 (2) (1994) 223-273. 